\title{
TDP-43 immunohistochemistry reveals extensive neuritic pathology in FTLD-U: a Midwest-Southwest Consortium for FTLD study
}

\author{
Kimmo J. Hatanpaa, MD, PhD ${ }^{1}$, Eileen H. Bigio, $\mathrm{MD}^{2}$, Nigel J. Cairns, PhD, MRCPath ${ }^{3}, \mathrm{Kyle}$ \\ B. Womack, M.D., M.S. ${ }^{4}$, Sandra Weintraub, $\mathrm{PhD}^{5}$, John C. Morris, M.D. ${ }^{3}$, Chan Foong, \\ MS $^{1}$, Guanghua Xiao, PhD ${ }^{6}$, Christa Hladik ${ }^{1}$, Tina Y. Mantanona, BM ${ }^{1}$, and Charles L. White \\ III, MD ${ }^{1}$ \\ 1 Department of Pathology and Alzheimer's Disease Center, University of Texas Southwestern School of \\ Medicine, Dallas, $T X$
}

2 Department of Pathology and Alzheimer's Disease Center, Northwestern University Feinberg School of Medicine, Chicago, IL

3 Departments of Neurology and Pathology \& Immunology, and Alzheimer's Disease Research Center, Washington University School of Medicine, St. Louis, MO

4 Department of Neurology and Alzheimer's Disease Center, University of Texas Southwestern School of Medicine, Dallas, TX

5 Department of Cognitive Neurology and Alzheimer's Disease Center, Northwestern University Feinberg School of Medicine, Chicago, IL

6 Department of Clinical Sciences, University of Texas Southwestern School of Medicine, Dallas, TX

\section{Abstract}

TDP-43 is a major component of the inclusions in frontotemporal lobar degeneration with ubiquitinated inclusions (FTLD-U). We studied TDP-43-pathology in the hippocampus and frontal cortex of autopsy brains with FTLD-U $(n=68)$, dementia lacking distinctive histopathology $(n=4)$, other neurodegenerative diseases $(n=23)$, and controls $(n=12)$. A marked enhancement of TDP-43positive dystrophic neurites (DN) was obtained by using a sensitive immunohistochemistry protocol. Two previously unrecognized patterns of pathology were observed: frequent long DN in the CA1 region and frequent dot-like DN in the neocortical layer II, which were seen in 39\% and 15\% of the FTLD-U cases, respectively. Four FTLD-U cases showed no TDP-43 pathology and were reclassified as FTLD-U, non-TDP-43 proteinopathy. Frequent long DN, but not dot-like DN, were significantly associated with progranulin mutations. Three of the DLDH cases were reclassified as FTLD-U. Of the cases with other neurodegenerative diseases, $43 \%$ showed TDP-43-pathology in the hippocampus but only $4 \%$ in the frontal cortex. No TDP-43-pathology was seen in controls. These results indicate that the sensitivity of the TDP-43 immunohistochemistry method affects both the quantity of the pathology and the types of pathology that can be detected. Involvement of both the hippocampus and frontal cortex may be a diagnostically important feature in FTLD-U.

Corresponding author: Kimmo Hatanpaa, MD, PhD; H2.130, 5323 Harry Hines Blvd, Department of Pathology, University of Texas Southwestern School of Medicine, Dallas, TX 75390. Phone 214-648-2148; fax 214-648-2077. E-mail:

Kimmo.Hatanpaa@UTSouthwestern.edu. 


\section{Keywords}

Frontotemporal lobar degeneration with ubiquitinated inclusions; frontotemporal lobar degeneration with motor neuron disease; dementia lacking distinctive histopathology; progranulin; TAR DNAbinding protein 43; dystrophic neurites; autopsy; immunohistochemistry

\section{INTRODUCTION}

In 1996, Jackson, Lennox, and Lowe described a series of patients with frontotemporal dementia (FTD) and atrophy of the frontal and temporal lobes (1). None of the patients had clinical evidence of either an upper or lower motor neuron disorder. In each case, brain autopsy revealed neuronal inclusions identical to those described previously in dementia associated with motor neuron disease (amyotrophic lateral sclerosis with dementia). Originally named as motor neuron disease inclusion dementia and believed to be rare, this disease is now referred to as frontotemporal lobar degeneration with ubiquitinated inclusions (FTLD-U) (2) and is known to be the most common cause of frontotemporal dementia (3). The pathologic hallmarks of FTLD-U are neuronal cytoplasmic inclusions, dystrophic neurites, and occasional neuronal intranuclear inclusions, all of which are immunoreactive with antibodies to the relatively nonspecific marker ubiquitin and generally negative for other proteins such as tau, $\alpha$-synuclein, $\mathrm{A} \beta, \alpha$-internexin, and polyglutamine $(4,5)$.

Recently, TAR DNA binding protein 43 (TDP-43), a nuclear protein involved in exon skipping and transcription regulation, was identified as a major component of the inclusions and dystrophic neurites in FTLD-U, FTLD-U with motor neuron disease, and amyotrophic lateral sclerosis (4). Another major recent advance was the discovery that autosomal dominant null mutations in the progranulin gene are the genetic basis for most cases of familial FTLD-U linked to chromosome 17 (6-9).

Dementia lacking distinctive histopathology (DLDH) and the closely related hippocampal sclerosis dementia (HSD) are terms used for cases of dementia where no inclusions are found at autopsy to allow further pathological classification (10-13). Frontal or temporal lobe atrophy and neuron loss are usually present in DLDH, whereas pronounced hippocampal atrophy and neuron loss are seen in HSD. FTD is the most common clinical presentation $(10,14)$. DLDH was previously thought to be the most common type of pathology underlying FTD, but studies using ubiquitin immunohistochemistry have demonstrated that most cases of DLDH and HSD represent FTLD-U $(3,15,16)$. The term hippocampal sclerosis refers simply to a severe loss of neurons in the hippocampal pyramidal cell layer; hippocampal sclerosis can be associated with many neurodegenerative diseases, including Alzheimer's disease, although it is particularly common in FTLD-U (17).

In this study, we used a highly sensitive immunohistochemistry method and were able to increase and enhance the visible TDP-43 pathology significantly compared with the previously described routine TDP-43 immunohistochemistry protocol. We then combined all available autopsy brains with FTLD-U and DLDH from three centers and used the enhanced immunohistochemistry protocol to characterize TDP-43-pathology in the hippocampus and frontal cortex of these cases as well as in two types of control groups: cases with other neurodegenerative diseases and nondemented controls. Specifically, we used the enhanced immunohistochemistry method to determine the prevalence of TDP-43-pathology among our FTLD-U cases and to investigate the specificity of TDP-43 pathology for FTLD-U. In addition, we wanted to know if the types and severity of TDP-43 pathology differed between FTLD-U cases with progranulin mutations and FTLD-U cases without progranulin mutations. We also 
investigated whether any of the DLDH cases could be reclassified as FTLD-U based on the presence of TDP-43-pathology.

\section{MATERIALS AND METHODS}

\section{Case Material}

Autopsy brains with a diagnosis of either FTLD-U ( $n=68)$ or DLDH $(n=4)$ were selected from three centers based on availability (Table 1). All elderly control brains without history of dementia or neurodegenerative diseases $(n=12)$ were selected from one institution (UTSW). The group of other neurodegenerative diseases $(n=23)$ was selected with the aim to include a broad range of diagnoses and consisted of cases with the following diagnoses: Alzheimer's disease ( $A D ; n=8)$, Lewy body variant of $A D(n=1)$, neurofibrillary tangle dementia $(n=3)$, vascular dementia $(n=1)$, sporadic multiple system tauopathy with dementia $(2)(n=1)$, basophilic inclusion body disease $(n=1)$, FTLD with Pick bodies $(2)(n=3)$, progressive supranuclear palsy $(n=4)$, and cerebral autosomal dominant arteriopathy with subcortical infarcts and leukoencephalopathy (CADASIL; $n=1$ ). Diagnostic workup for these cases was performed according to published criteria (2) and included examination of $\mathrm{H} \& \mathrm{E}$ sections, thioflavin $S$ preparations, and immunohistochemical stains for $\alpha$-synuclein in all cases. Immunohistochemistry for phosphorylated tau, $A \beta$, and ubiquitin was performed in all cases of FTLD-U. Where indicated, immunohistochemistry was also been performed for $\alpha$ internexin, polyglutamine, $3 \mathrm{R}$ tau, $4 \mathrm{R}$ tau, and Notch3. The diagnosis of $\mathrm{AD}$ was based on tangle and plaque densities meeting the CERAD "definite" (18) and NIA-Reagan Institute "high likelihood" (19) criteria. Two cases of FTLD-U with VCP mutation, a very rare type of FTLD, were available. Because of the small number of FTLD-U cases with VCP mutations and the previous evidence that these cases represent their own frontal pathology subtype (subtype 4) (20), these cases were included in the analyses of the pathologic subtypes (Tables 5-6) but excluded from other analyses. A signed consent for brain autopsy had been obtained from the next of kin or legal representative in each case.

\section{Sequencing of the Progranulin Gene}

High molecular weight DNA was extracted from blood or frozen brain tissue of 58 cases according to standard procedures. The $P G R N$ gene was sequenced as previously described $(8,9)$.

\section{Antibodies for immunohistochemistry}

The primary antibodies were rabbit polyclonal antibodies for TDP-43 at a dilution of 1:1,000 (ProteinTech, Chicago, IL) and ubiquitin at a dilution of 1:500 (Dako, Carpinteria, CA). A mouse monoclonal TDP-43 antibody (2E2-D3; Novus Biologicals, Littleton, CO) was used in some cases for verification purposes.

\section{Routine Immunohistochemistry Protocol}

Formalin-fixed, paraffin-embedded tissue blocks from the posterior hippocampus at the level of the lateral geniculate nucleus and from the middle frontal gyrus were used. As previously described $(4,21)$, the slides underwent a pretreatment step with formic acid $(5 \mathrm{~min})$, the detection system was the avidin-biotin system, the chromogen was diaminobenzidine (DAB), and the procedure was performed manually. Ubiquitin immunohistochemistry which served as the basis of the original FTLD-U diagnoses was performed according to this routine method.

\section{Enhanced Immunohistochemistry Protocol}

In the enhanced immunohistochemistry protocol for TDP-43, the detection system was the alkaline phosphatase-based ultraView Red detection system (Ventana Medical Systems, 
Tucson, AZ), the chromogen was Fast Red/Naphthol, and the procedure was performed using the Benchmark XT automated stainer (Ventana). This automated stainer is commercially available, and more than 5,500 units are currently in use worldwide (Don Green, Ventana, personal communication, 9/24/2007). No pretreatment was found to be necessary. Ubiquitin immunohistochemistry using the enhanced protocol was not systematically performed or studied on all cases, and the results of ubiquitin immunohistochemistry in this paper refer to only the routine method.

\section{Evaluation of the Immunohistochemical Staining}

The results were evaluated semiquantitatively using a scale of $0-3$, where 0 corresponds to "none" and 3 corresponds to "frequent". The evaluator was a neuropathologist $(\mathrm{KJH})$ blinded to clinical data and diagnoses.

\section{Double Labeling Immunohistochemistry}

Double labeling immunohistochemistry for phosphorylated tau and TDP-43 was performed in selected cases using the Benchmark XT automated stainer. After pretreatment with the Cell Conditioning Solution (Ventana), the sections were exposed to a mouse monoclonal PHF antibody (AT8, Innogenetics, Ghent, Belgium) at a dilution of 1:200. The detection system was the ultraView Universal DAB and the chromogen was DAB. The sections were then processed for TDP-43 immunohistochemistry as described above.

\section{FTLD-U frontal cortex pathology subtypes}

FTLD-U frontal cortex pathology subtypes were determined as previously described (20).

\section{Statistics}

Comparisons of frequency data were performed using Fisher's exact probability test (22). Student's t-test assuming unequal variances was used for comparisons of continuous variables. All tests were two-tailed. The level of statistical significance was set at $\mathrm{p}=0.05$.

Statistical tests involving the dystrophic neurites variable, originally collected as a four-class ordered category variable (none, sparse, moderate, frequent), were performed by two alternative methods, which, as it turned out, produced very similar results. The first method consisted of dichotomization of the variable into two classes (none to moderate and frequent), followed by Fisher's exact probability test. This approach was chosen because of its simplicity and because of our research question, which was to compare the frequent category to all other categories. In addition, we observed that the dystrophic neurites variable followed a nearly dichotomous distribution in the hippocampus, i.e., cases falling into the moderate category were rare. A second, alternative statistical approach was also undertaken to deflect any possible criticism suggesting that the dichotomization may have been too arbitrary and that it may have led to loss of information. The second method consisted of the Mantel Haenszel Chi Square Test (23), performed using the SAS software (SAS Institute Inc., Cary, North Carolina). All of the original category data were used in this test. As shown in Results, the p-values that were obtained with the second approach were similar to those obtained with the first approach. In addition, all p-values that were significant with the first method remained significant with the second method, and all p-values that were nonsignificant with the first method remained nonsignificant with the second method.

Agreement of frontal pathology subclassification based on routine and enhanced immunohistochemistry protocols was evaluated using Cohen's unweighted kappa (22). 


\section{RESULTS}

\section{Increased Sensitivity of the Immunohistochemistry Method}

In some FTLD-U cases, use of the enhanced immunohistochemistry method lead to dramatic increases in the staining intensity and frequency of visible TDP-43-positive dystrophic neurites compared with the routine method. The difference was particularly striking in the hippocampal CA1 region, where the dystrophic neurites were strongly positive and frequent in $39 \%$ of the FTLD-U cases by the enhanced method (Fig. 1). The area of frequent dystrophic neurites was typically well demarcated and limited to the CA1 region (Fig. 2), in some cases also involving the prosubiculum. In contrast, routine immunohistochemistry revealed either no dystrophic neurites at all or weakly positive, sparse dystrophic neurites in CA1 (Fig 1A). Similar results were obtained with the routine method performed at two centers. The enhanced method did not result in significant increases in the staining intensity or frequency of cytoplasmic inclusions or intranuclear inclusions. The nondemented control cases remained negative for TDP-43 pathology even with the enhanced method. Unless specified otherwise, further analyses were performed using data only from the enhanced immunohistochemistry method.

\section{Prevalence of TDP-43-pathology}

Three of the four DLDH cases showed TDP-43 pathology both in the hippocampus and frontal cortex and were reclassified as FTLD-U (Table 1). No TDP-43 pathology was found in the one remaining DLDH case. Of the cases with other neurodegenerative diseases, $43 \%$ showed TDP-43-pathology in the hippocampus but only $4 \%$ (a single case) in the frontal cortex. In contrast, 97\% of FTLD-U cases had TDP-43-pathology in both the hippocampus and the frontal cortex. No TDP-43-pathology was seen in the controls without neurodegenerative diseases or history of dementia. Four FTLD-U cases (6\%) failed to show any TDP-43 pathology by immunohistochemistry using either the polyclonal or monoclonal TDP-43 antibody and were reclassified as FTLD-U, non-TDP-43 proteinopathy (Fig. 3).

\section{Dystrophic Neurites in CA1}

The frequency of TDP-43-positive dystrophic neurites in the hippocampal CA1 region of FTLD-U cases showed a dichotomous distribution with a large majority of cases showing either no to sparse neurites (55\%) or frequent neurites (39\%). Moderate numbers of dystrophic neurites were uncommon in CA1 (6\%). Similar dichotomy was not observed in the frequency distribution of the cytoplasmic inclusions in the hippocampal dentate gyrus or the frequency distribution of dystrophic neurites in the frontal cortex, where $32 \%$ and $27 \%$ of cases, respectively, fell into the moderate category.

Frequent TDP-43-positive dystrophic neurites in the CA1 region were significantly associated with progranulin mutations (Table 2). Progranulin mutations were found in $60 \%$ of the cases with frequent dystrophic neurites in CA1 and in $17 \%$ of the cases with none to moderate dystrophic neurites in CA1 (Fisher's exact probability test $p=0.007$, Mantel Haenszel Chi Square Test $\mathrm{p}=0.001$ ).

For verification purposes, the hippocampi of two FTLD-U cases with frequent dystrophic neurites in CA1 were also stained using the mouse monoclonal TDP-43 antibody. A similar pattern of frequent dystrophic neurites was observed with the monoclonal TDP-43 antibody, although the staining intensity was weaker compared with the polyclonal antibody.

\section{Dystrophic Neurites in the Frontal Cortex}

In the frontal cortex, two types of TDP-positive dystrophic neurites were seen: long dystrophic neurites, which were similar to the dystrophic neurites in CA1, and distinctive, dot-like dystrophic neurites (Fig. 4). Both types of neurites were typically most frequent in layer II, 
although the long neurites were generally more widely dispersed throughout the entire thickness of the cortex compared with the dot-like neurites. Frequent long dystrophic neurites were present in the frontal cortex in 35\% of FTLD-U cases and in either frontal cortex or CA1 in $50 \%$ of FTLD-U cases.

Frequent TDP-43-positive, long dystrophic neurites in the frontal cortex were significantly associated with progranulin mutations (Table 3). Progranulin mutations were found in $71 \%$ of FTLD-U cases with frequent long neurites and in $4 \%$ of FTLD-U cases with none to moderate long neurites in the frontal cortex (Fisher's exact probability test $p=0.00008$, Mantel Haenszel Chi Square Test $p<0.0001$ ). Solid inclusions (Fig. 5) were the predominant type of cytoplasmic inclusions in $100 \%$ of FTLD-U cases with frequent long neurites and in 56\% of cases with none to moderate long neurites in the frontal cortex (Fisher's exact probability test $\mathrm{p}=0.0003$, Mantel Haenszel Chi Square Test $\mathrm{p}<0.0001)$.

Frequent TDP-43-positive, dot-like dystrophic neurites were seen in an additional 15\% of FTLD-U cases in the frontal cortex. Unlike frequent long neurites, the dot-like neurites were not significantly associated with progranulin mutations and were associated with granular rather than solid cytoplasmic inclusions in the frontal cortex (Table 4). Granular inclusions (Fig. 5) were the predominant cytoplasmic inclusion type in 67\% of FTLD-U cases with frequent dot-like neurites and in $20 \%$ of FTLD-U cases without frequent dot-like inclusions $(\mathrm{p}=0.01)$.

\section{Localization of TDP-43-pathology Relative to Tau-pathology}

Double labeling immunohistochemistry for phosphorylated tau and TDP-43 was performed on a case of neurofibrillary tangle dementia with coexistent TDP-43 pathology in the hippocampus (Fig. 6). The tau-positive tangles and TDP-43-inclusions appeared to be independent, as some neurons contained inclusions composed of tau only or TDP-43 only.

\section{FTLD-U Frontal Cortex Pathology Subtypes}

A preliminary analysis comparing frontal pathology subtypes (20) based on routine TDP-43 immunohistochemistry and routine ubiquitin immunohistochemistry showed that the subtypes were identical in all FTLD-U cases where both routine TDP-43 immunohistochemistry and routine ubiquitin immunohistochemistry were available $(n=36)$. Because routine ubiquitin immunohistochemistry on frontal cortex was available on a larger number of cases $(n=63)$ than routine TDP-43 immunohistochemistry, the data on routine ubiquitin immunohistochemistry was selected for further analysis, whereas enhanced TDP-43 immunohistochemistry was used to represent enhanced immunohistochemistry. Comparison of the number of cases falling into each based on the routine and enhanced immunohistochemistry methods showed that there was substantial agreement between the two methods among the cases classifiable by both methods (kappa $=0.61$ ); however, 10 cases were unclassifiable by the enhanced method because of the presence of frequent dot-like dystrophic neurites in the frontal cortex (Table 5). Frequent dystrophic neurites in the CA1 region of the hippocampus were more common in subtype 3 compared with the other subtypes ( $\mathrm{p}=0.0007$; Table 6 ).

\section{TDP-43-pathology and Hippocampal Sclerosis}

Among the FTLD-U cases, the prevalence of hippocampal sclerosis, including severe neuronal loss limited to the CA1/subiculum border zone (24), was $25 \%$ in the posterior hippocampus at the level of the lateral geniculate nucleus and 57\% in the anterior hippocampus at the level of the pes hippocampi ( $\mathrm{p}=0.003$; Table 6 ). Hippocampal sclerosis was not disproportionally common in any particular FTLD-U frontal pathology subtype. The anterior hippocampus was available for examination from only 30 FTLD-U cases, which may have limited the evaluation 
for a possible correlaton between hippocampal sclerosis in the anterior hippocampus and the FTLD-U frontal cortex pathology subtypes.

\section{FTLD-U, non-TDP-43 Proteinopathy}

A minority of FTLD-U cases $(n=4)$ showed inclusions and dystrophic neurites by ubiquitin immunohistochemistry but not by routine or enhanced TDP-43 immunohistochemistry. These four cases were reclassified as FTLD-U, non-TDP-43 proteinopathy. Except for the lack of TDP-43-immunopositive inclusions and neurites, the clinical and pathologic features of the four cases overlapped with those seen in FTLD-U cases with TDP-43-proteinopathy (FTLD$\mathrm{U}$ ) (Table 7). However, the average age of onset was significantly younger in the four cases (42 years) compared with the other FTLD-U cases (59 years, $\mathrm{p}=0.006$ ). Two of the four cases were tested for progranulin mutations and both were found to be negative.

\section{DISCUSSION}

It can be hypothesized that the TDP-43-positive neuritic pathology in FTLD-U, possibly due to its potential to directly interfere with synaptic function, may turn out to be the closest pathologic correlate of clinical dementia. These results show that FTLD-U is characterized by more extensive TDP-43-positive neuritic pathology than what has been previously described. The full extent of the neuritic pathology can be visualized by increasing the sensitivity of the immunohistochemistry protocol (Fig. 1).

In this study, the increased sensitivity over the routine avidin-biotin immunohistochemistry method was achieved by using a commercially available automated stainer, a detection system that contains an amplification step, and a high-contrast red chromogen. Several other highly sensitive immunohistochemistry methods are available $(25,26)$. These methods do not require an automated stainer. They have been shown to be over 10 times more sensitive than the avidinbiotin method. It is possible that these other sensitive immunohistochemistry methods would have produced results similar to those presented here.

Mackenzie et al. performed ubiquitin immunohistochemistry on cases previously diagnosed as dementia lacking distinctive histopathology (DLDH) using a similar automated stainer but with a different detection system. The authors reported increased numbers of ubiquitin-positive cytoplasmic inclusions in the dentate gyrus and increased numbers of dystrophic neurites in the frontal and temporal cortex (16).

The preferential enhancement of certain types of TDP-43-pathology (neurites vs. cytoplasmic inclusions) and certain anatomic areas (CA1 vs. neocortex) by the more sensitive immunohistochemistry method is consistent with biochemical heterogeneity of TDP-43pathology. Posttranslational modifications such as phosphorylation and truncation, previously reported to occur in TDP-43-pathology (4), may cause variability in the affinity of the TDP-43 antibody for its antigen.

The distribution of frequent TDP-43-positive dystrophic neurites in the hippocampus (CA1 or CA1-prosubiculum) in FTLD-U, as revealed by this study, corresponds closely to the area typically affected by the type of severe neuronal loss known as hippocampal sclerosis. Given the similar anatomical distribution of frequent TDP-43-positive dystrophic neurites and hippocampal sclerosis within the hippocampus of FTLD-U cases, it can be hypothesized that frequent dystrophic neurites may represent a precursor lesion of hippocampal sclerosis.

Hippocampal sclerosis can be seen in many neurodegenerative diseases, but it is particularly common in FTLD-U $(15,27)$. In this study, the prevalence of hippocampal sclerosis among the FTLD-U cases was $42 \%$. An additional finding supporting the hypothesis that frequent dystrophic neurites may represent the precursor lesion of hippocampal sclerosis is the 
dichotomous severity distribution of the dystrophic neurites observed in this study: in the large majority of FTLD-U cases (94\%), the dystrophic neurites in the CA1 region were either absent/ sparse or very frequent. Neuronal loss in the CA1 region follows a similar dichotomous severity distribution in FTLD-U, being either unrecognizable/very mild or very severe (hippocampal sclerosis) in a large majority of cases (unpublished observation).

Frequent TDP-43-positive, long dystrophic neurites in CA1 and frontal cortex were significantly associated with progranulin mutations, whereas no such association was found between frequent dot-like dystrophic neurites in the frontal cortex and progranulin mutations (Tables 2-4, Fig. 4). With regard to the long dystrophic neurites, our results are consistent with those of Josephs et al, who used ubiquitin immunohistochemistry and showed a greater density of ubiquitin-positive dystrophic neurites in the neocortex of FTLD-U cases with progranulin mutations compared with cases without progranulin mutations (26). Cairns et al., using the avidin-biotin immunohistochemistry protocol, reported a strong association between progranulin mutations and type 3 pattern of neocortical TDP-43-pathology, which was defined as "pathology predominantly in the superficial cortical layers with numerous neuronal cytoplasmic inclusions, dystrophic neurites, and variable numbers of neuronal intranuclear inclusions", whereas no association was found between progranulin mutations and type 1 pattern, which was defined as "abundance of long dystrophic neurites predominantly in superficial cortical laminae, with few or no neuronal cytoplasmic inclusions or neuronal intranuclear inclusions" (20). In the present study, where the enhanced immunohistochemistry method was used, all cases with frequent long dystrophic neurites in the frontal cortex also had at least moderate cytoplasmic inclusions. Thus, the cases with frequent long dystrophic neurites in the frontal cortex would probably correspond to type 3 in the classification of Cairns et al. and would be unclassifiable in an earlier version of the same classification, where the type 3 pattern included the requirement of "short neuritic profiles" (21). The cases with frequent dotlike neurites would be unclassifiable by both classification systems. When the criteria of Cairns et al. were used and the unclassifiable cases were excluded, there was substantial agreement between the routine and enhanced immunohistochemistry methods (Table 5).

Four FTLD-U cases (6\%) did not show any TDP-43-positive pathology although ubiquitinpositive inclusions were present (Fig. 3, Table 7). The ages at onset in these unrelated patients were $33,39,54$, and 59 years, respectively, whereas the average age at onset among the other FTLD-U patients was 59 years $(\mathrm{p}=0.006)$. A young age of onset may suggest a genetic component in the pathogenesis. Recently, a mutation in the charged multivesicular body protein 2B (CHMP2B) gene in chromosome 3 was shown to be the cause of FTLD-U, non TDP-43 proteinopathy in a large Danish pedigree $(20,28)$. The CHMP2B mutation status of our four cases is currently unknown. Progranulin mutation screening has been performed in two of the cases. Both cases were negative for progranulin mutations.

Of the four cases originally classified as DLDH based on a diagnostic workup that included ubiquitin immunohistochemistry by the routine method, only one case remained as DLDH after TDP-43 immunohistochemistry by the enhanced method. The patient died at age 98 after a 10-year clinical history of Alzheimer-like dementia. Further review of the pathologic findings in this one remaining DLDH case raised the possibility of vascular dementia as an alternative diagnosis.

Among the cases with other neurodegenerative diseases, $35 \%$ showed mild to moderate TDP-43 pathology in the hippocampus but not in the frontal cortex. In contrast, 97\% of FTLD$\mathrm{U}$ cases had TDP-43-pathology in both the hippocampus and frontal cortex. Our results are in line with a recent report of TDP-43-pathology in the hippocampus of 20\% of Alzheimer's disease cases (29). 
The specificity of the TDP-43 staining observed with the enhanced method is supported by a number of findings, including the following: nondemented controls did not have any TDP-43 pathology, TDP-43 pathology was anatomically restricted in cases with other neurodegenerative diseases, results obtained with two different TDP-43 antibodies showed a similar pattern of staining, nuclear staining was always present as an internal positive control, the presence of frequent long neurites in the CA1 and frontal cortex correlated with progranulin mutations (Tables 2-3), substantial agreement was found between the routine and enhanced protocols in the frontal cortex when the cases with dot-like neurites were excluded (Table 5), and the presence of frequent dot-like neurites in the frontal cortex correlated with granular cytoplasmic inclusions and younger age of the patients (Table 4).

These results demonstrate that the sensitivity of the immunohistochemistry protocol can have a pronounced effect on the number of visible TDP-43-positive neurites in FTLD-U. Extensive neuritic pathology is common in the hippocampal CA1 region and forms a well-demarcated, previously unrecognized type of lesion that is readily identifiable only when a sensitive immunohistochemistry protocol is used. Interestingly, this pattern of pathology is usually either clearly present (visible even without a microscope) or absent -cases falling into the "moderate" category were rare. Frequent TDP-43-positive, dystrophic neurites in the CA1 region and frontal cortex are associated with progranulin mutations. However, frequent dot-like like neurites in the frontal cortex showed no such association. Although the presence of dystrophic neurites in FTLD-U was previously known, the patterns of pathology (frequent CA1 neurites and dot-like neurites) described here are novel and their identification was made possible by the increased sensitivity of the detection method.

\section{Acknowledgements}

We thank Drs. Alison Goate (Washington University School of Medicine, St. Louis, MO) and Mike Hutton (Mayo Clinic College of Medicine, Jacksonville, FL) for providing the progranulin sequencing data, Dr. Krystyna R. Isaacs for critical review of the manuscript, and Ms. Ping Shang for expert technical assistance.

Supported by NACC 2005-05, NIH AG12300, P50-AG05681, P01-AG03991, P30 AG013854, Winspear Family Center for Research on the Neuropathology of Alzheimer Disease, and McCune Foundation.

\section{References}

1. Jackson M, Lennox G, Lowe J. Motor neurone disease-inclusion dementia. Neurodegeneration 1996;5:339-50. [PubMed: 9117546]

2. Cairns NJ, Bigio EH, Mackenzie IR, et al. Neuropathologic diagnostic and nosologic criteria for frontotemporal lobar degeneration: consensus of the Consortium for Frontotemporal Lobar Degeneration. Acta Neuropathol (Berl) 2007;114:5-22. [PubMed: 17579875]

3. Lipton AM, White CL 3rd, Bigio EH. Frontotemporal lobar degeneration with motor neuron diseasetype inclusions predominates in 76 cases of frontotemporal degeneration. Acta Neuropathol (Berl) 2004;108:379-85. [PubMed: 15351890]

4. Neumann M, Sampathu DM, Kwong LK, et al. Ubiquitinated TDP-43 in frontotemporal lobar degeneration and amyotrophic lateral sclerosis. Science 2006;314:130-3. [PubMed: 17023659]

5. Davidson Y, Kelley T, Mackenzie IR, et al. Ubiquitinated pathological lesions in frontotemporal lobar degeneration contain the TAR DNA-binding protein, TDP-43. Acta Neuropathol (Berl) 2007;113:521-33. [PubMed: 17219193]

6. Baker M, Mackenzie IR, Pickering-Brown SM, et al. Mutations in progranulin cause tau-negative frontotemporal dementia linked to chromosome 17. Nature 2006;442:916-9. [PubMed: 16862116]

7. Cruts M, Gijselinck I, van der Zee J, et al. Null mutations in progranulin cause ubiquitin-positive frontotemporal dementia linked to chromosome 17q21. Nature 2006;442:920-4. [PubMed: 16862115]

8. Gass J, Cannon A, Mackenzie IR, et al. Mutations in progranulin are a major cause of ubiquitin-positive frontotemporal lobar degeneration. Hum Mol Genet 2006;15:2988-3001. [PubMed: 16950801] 
9. Mukherjee O, Pastor P, Cairns NJ, et al. HDDD2 is a familial frontotemporal lobar degeneration with ubiquitin-positive, tau-negative inclusions caused by a missense mutation in the signal peptide of progranulin. Ann Neurol 2006;60:314-22. [PubMed: 16983685]

10. Giannakopoulos P, Hof PR, Bouras C. Dementia lacking distinctive histopathology: clinicopathological evaluation of 32 cases. Acta Neuropathol (Berl) 1995;89:346-55. [PubMed: 7610766]

11. Knopman DS, Mastri AR, Frey WH 2nd, et al. Dementia lacking distinctive histologic features: a common non-Alzheimer degenerative dementia. Neurology 1990;40:251-6. [PubMed: 2300243]

12. Corey-Bloom J, Sabbagh MN, Bondi MW, et al. Hippocampal sclerosis contributes to dementia in the elderly. Neurology 1997;48:154-60. [PubMed: 9008511]

13. Jellinger KA. Hippocampal sclerosis: a common pathological feature of dementia in very old humans. Acta Neuropathol (Berl) 1994;88:599. [PubMed: 7879610]

14. Blass DM, Hatanpaa KJ, Brandt J, et al. Dementia in hippocampal sclerosis resembles frontotemporal dementia more than Alzheimer disease. Neurology 2004;63:492-7. [PubMed: 15304580]

15. Hatanpaa KJ, Blass DM, Pletnikova O, et al. Most cases of dementia with hippocampal sclerosis may represent frontotemporal dementia. Neurology 2004;63:538-42. [PubMed: 15304590]

16. Mackenzie IR, Shi J, Shaw CL, et al. Dementia lacking distinctive histology (DLDH) revisited. Acta Neuropathol (Berl) 2006;112:551-9. [PubMed: 16900341]

17. Josephs KA, Dickson DW. Hippocampal sclerosis in tau-negative frontotemporal lobar degeneration. Neurobiol Aging 2007;28:1718-22. [PubMed: 16930776]

18. Mirra SS, Hart MN, Terry RD. Making the diagnosis of Alzheimer's disease. A primer for practicing pathologists. Arch Pathol Lab Med 1993;117:132-44. [PubMed: 8427562]

19. Hyman BT, Trojanowski JQ. Consensus recommendations for the postmortem diagnosis of Alzheimer disease from the National Institute on Aging and the Reagan Institute Working Group on diagnostic criteria for the neuropathological assessment of Alzheimer disease. J Neuropathol Exp Neurol 1997;56:1095-7. [PubMed: 9329452]

20. Cairns NJ, Neumann M, Bigio EH, et al. TDP-43 in Familial and Sporadic Frontotemporal Lobar Degeneration with Ubiquitin Inclusions. Am J Pathol 2007;171:227-40. [PubMed: 17591968]

21. Sampathu DM, Neumann M, Kwong LK, et al. Pathological heterogeneity of frontotemporal lobar degeneration with ubiquitin-positive inclusions delineated by ubiquitin immunohistochemistry and novel monoclonal antibodies. Am J Pathol 2006;169:1343-52. [PubMed: 17003490]

22. Lowry, R. VassarStats: Web site for statistical computation. Vassar College; Poughkeepsie, NY: 1998.

23. Agresti, A. Introduction to categorical data analysis. New York: John Wiley and Sons; 1996.

24. Nakano I. Frontotemporal dementia with motor neuron disease (amyotrophic lateral sclerosis with dementia). Neuropathology 2000;20:68-75. [PubMed: 10935441]

25. Russo D, Ambrosino A, Vittoria A, et al. Signal amplification by combining two advanced immunohistochemical techniques. Eur J Histochem 2003;47:379-84. [PubMed: 14706935]

26. Qvigstad G, Sandvik AK, Brenna E, et al. Detection of chromogranin A in human gastric adenocarcinomas using a sensitive immunohistochemical technique. Histochem J 2000;32:551-6. [PubMed: 11127976]

27. Josephs KA, Dickson DW. Hippocampal sclerosis in tau-negative frontotemporal lobar degeneration. Neurobiol Aging. 2006

28. Skibinski G, Parkinson NJ, Brown JM, et al. Mutations in the endosomal ESCRTIII-complex subunit CHMP2B in frontotemporal dementia. Nat Genet 2005;37:806-8. [PubMed: 16041373]

29. Amador-Ortiz C, Lin WL, Ahmed Z, et al. TDP-43 immunoreactivity in hippocampal sclerosis and Alzheimer's disease. Ann Neurol 2007;61:435-45. [PubMed: 17469117] 


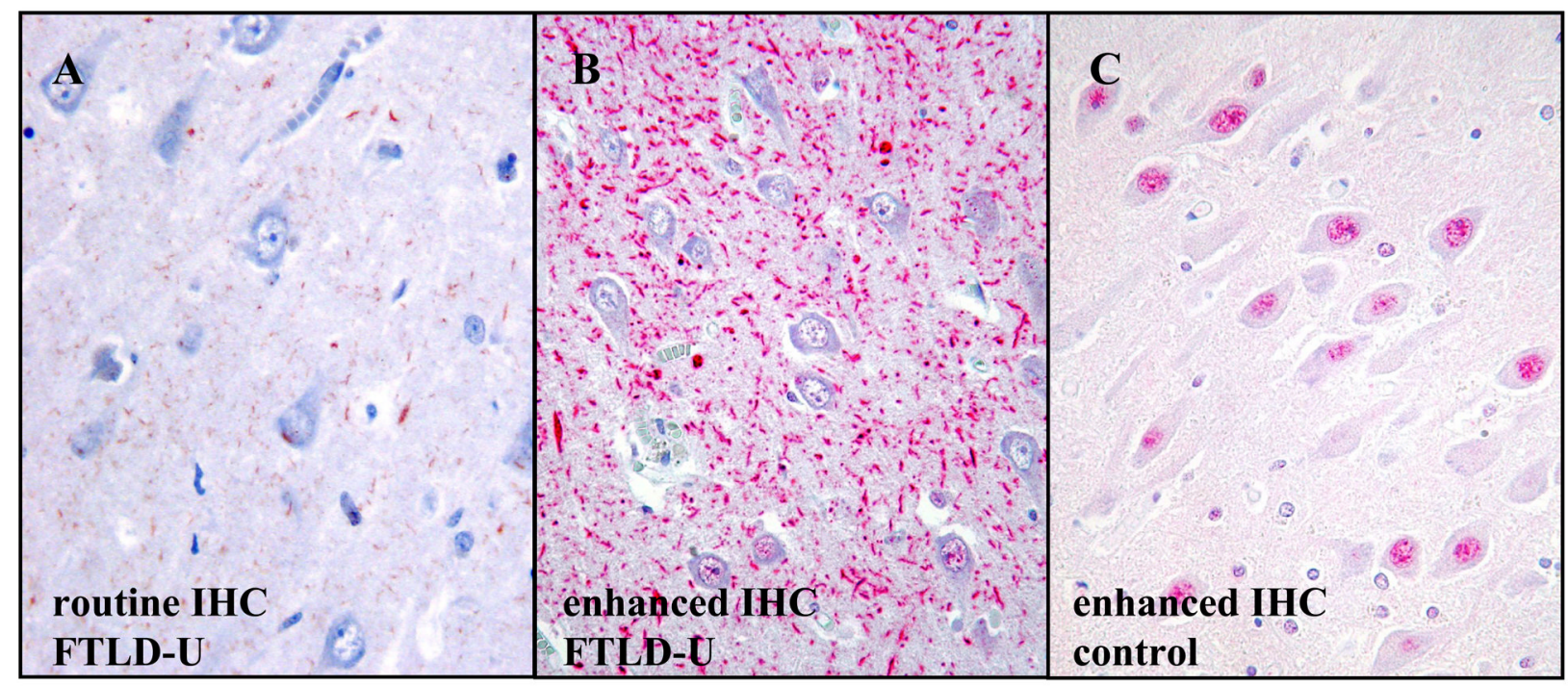

Figure 1.

Improvements in the immunohistochemistry (IHC) protocol lead to pronounced increases in visible TDP-43-pathology in some cases, particularly in the hippocampal CA1 region. (A) Routine immunohistochemistry for TDP-43 shows thin, weakly labeled dystrophic neurites (brown chromogen) in the CA1 region of an FTLD-U case. Comparable results were observed when the routine immunohistochemistry method was independently performed in another center. (B) In the same field of the same FTLD-U case, the enhanced TDP-43

immunohistochemistry method reveals frequent, strongly labeled neurites (red chromogen). Similarly frequent neurites were seen in the CA1 region of $39 \%$ of the FTLD-U cases. The dystrophic neurites are predominantly elongated rather than dot-like. Note that in both A and $\mathrm{B}$, the nuclei are negative, which is consistent with displacement of TDP-43 from the nucleus to the neurites. $(\mathbf{C})$ In a nondemented control case, the enhanced immunohistochemistry protocol shows nuclear positivity but no neurites. (Original magnification, 200X). 


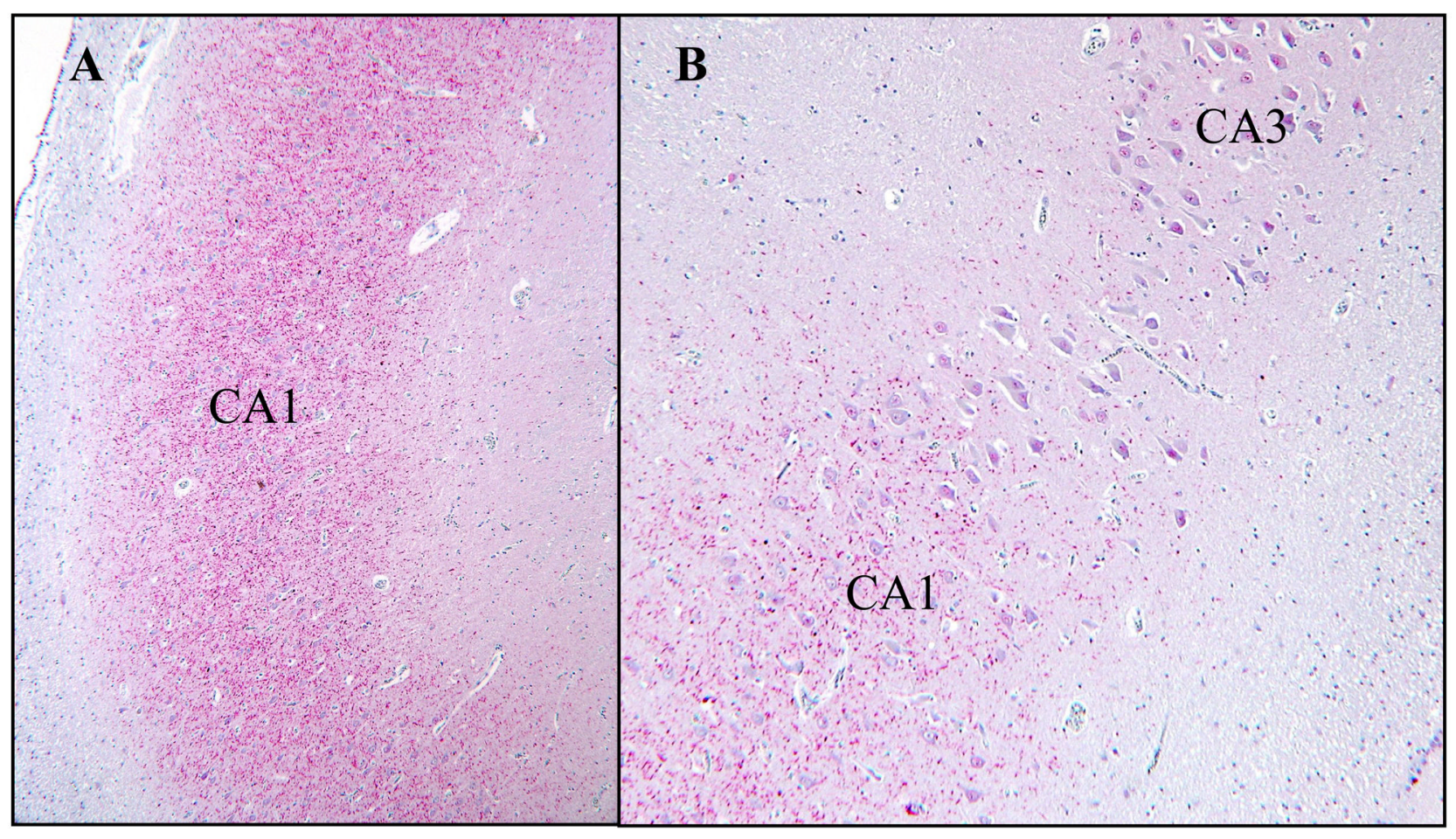

Figure 2.

Frequent TDP-43-positive dystrophic neurites in the CA1 region of an FTLD-U case. (A) At low magnification, the dystrophic neurites are evident as a dense band covering the CA1 region (40X). (B) The area of frequent dystrophic neurites is well demarcated and in most cases limited to CA1 (400X). 


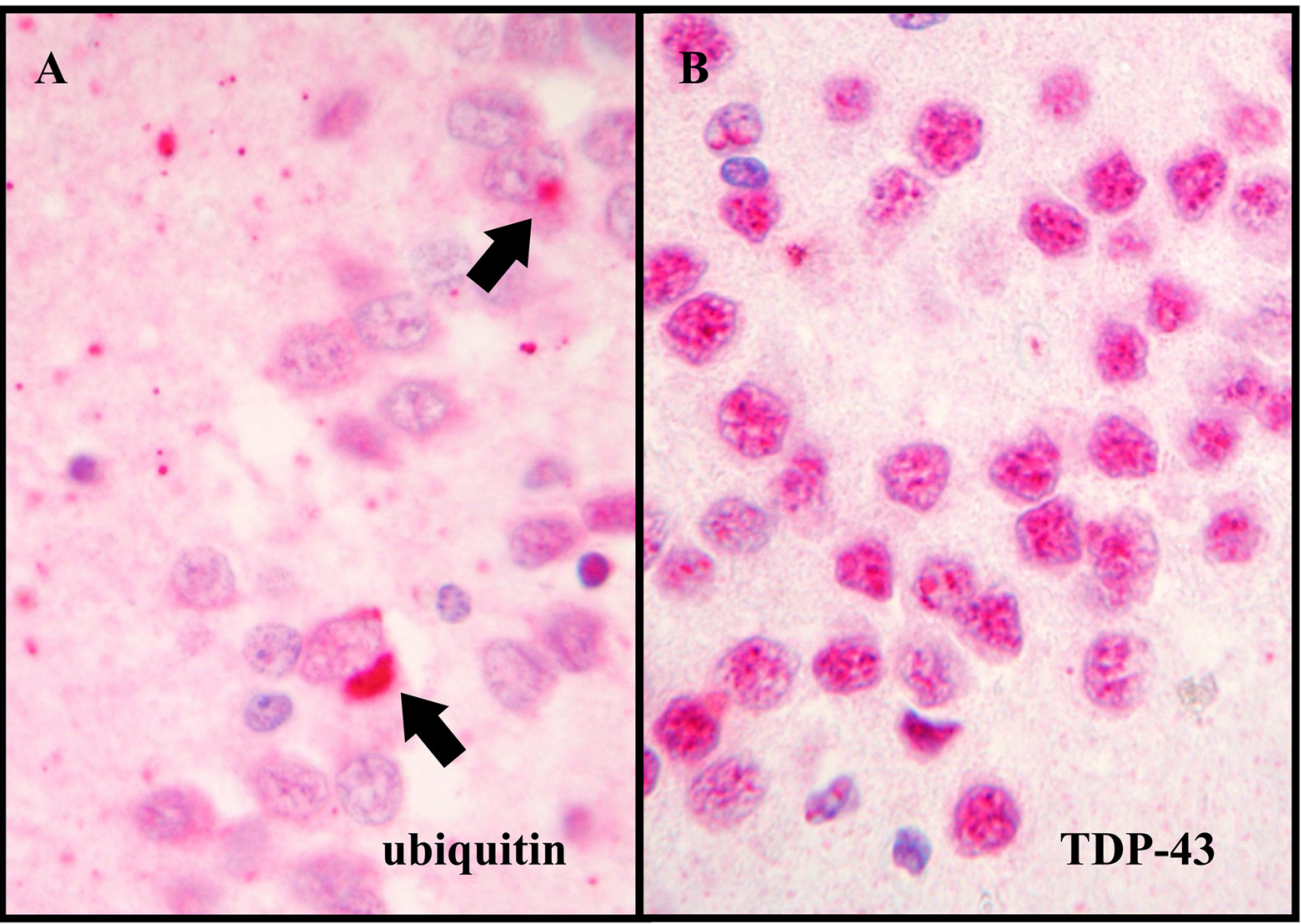

Figure 3.

An example of FTLD-U, non-TDP-43 proteinopathy. (A) Ubiquitin-positive neuronal cytoplasmic inclusions (arrows) in the dentate gyrus (400X; ubiquitin immunohistochemistry). (B) No inclusions are evident in the same area by TDP-43 immunohistochemistry (400X). 


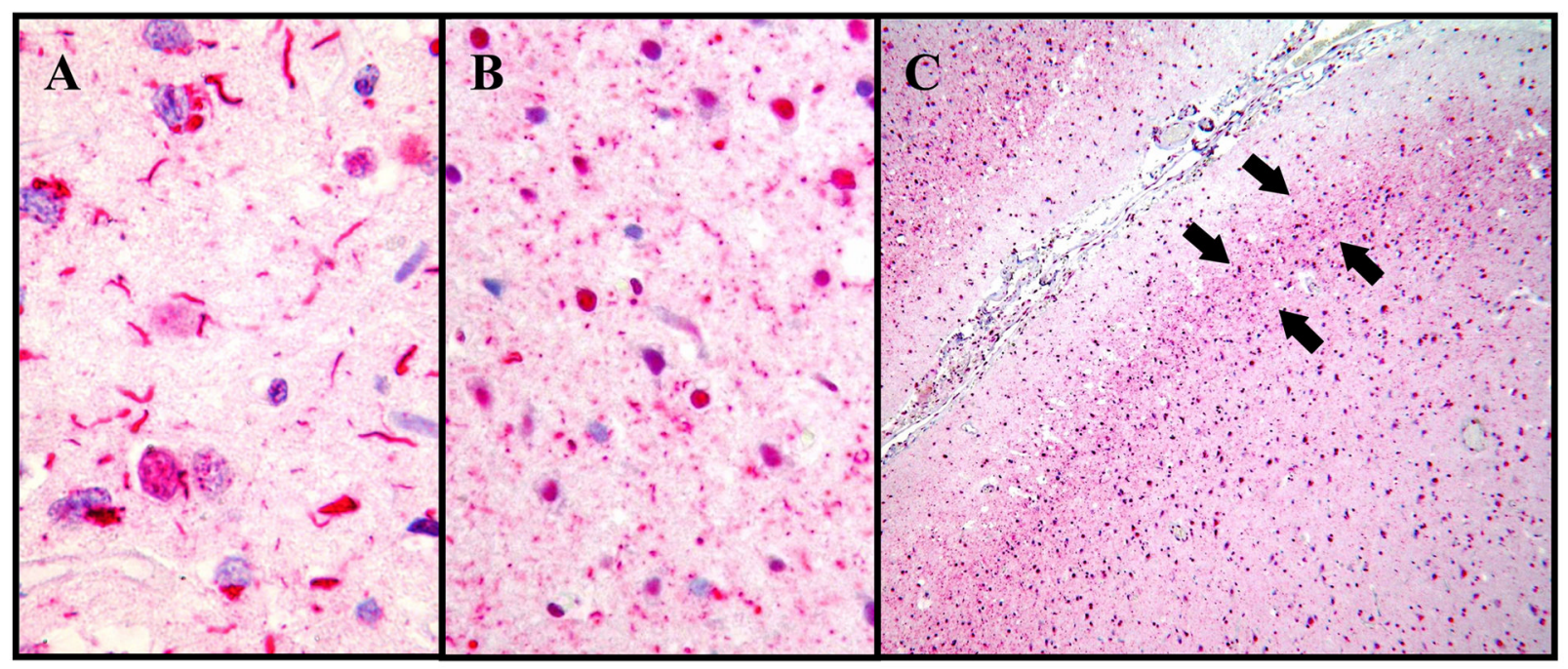

Figure 4.

Two types of TDP-43-positive dystrophic neurites were evident in the frontal cortex of FTLD$\mathrm{U}$ cases by immunohistochemistry. (A) Long neurites were frequent in the frontal cortex of $35 \%$ of FTLD-U cases (400X). (B) Dot-like neurites were frequent in an additional 14\% of FTLD-U cases (400X). (C) The dot-like neurites were concentrated in layer II (arrows; 40X). 


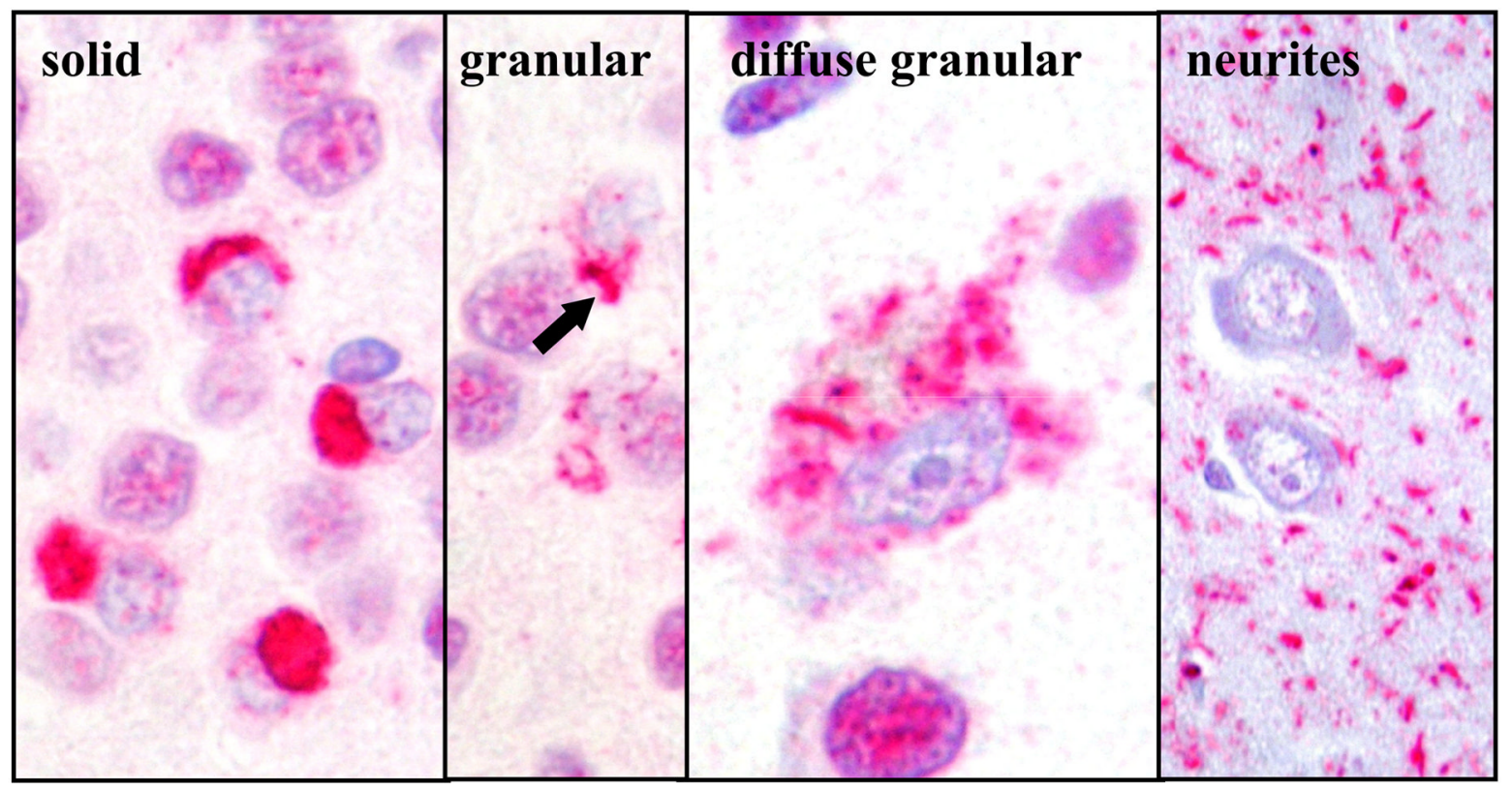

Figure 5.

Examples of TDP-43 inclusion types (TDP-43 immunohistochemistry, 400X). 


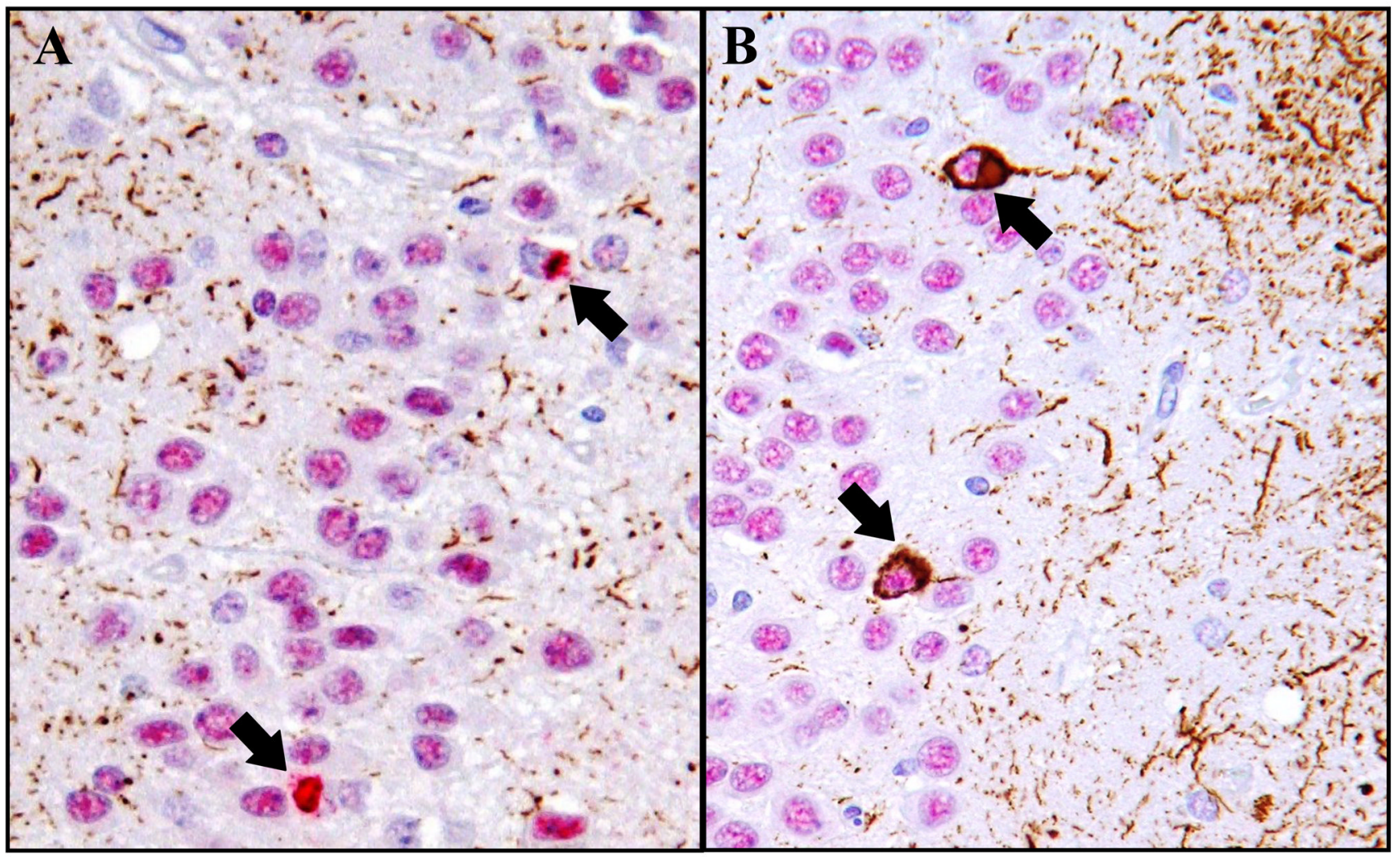

Figure 6.

Double labeling immunohistochemistry for phosphorylated tau (brown) and TDP-43 (red) in the dentate gyrus of a case with tangle predominant senile dementia and coexistent TDP-43 pathology. As in this example, coexistent TDP-43 pathology was seen in 35\% of the cases with other neurodegenerative diseases. It always involved the hippocampus and not the frontal cortex. (A) Cytoplasmic TDP-43-inclusions (arrows) are associated with loss of nuclear TDP-43-immunoreactivity due to displacement of TDP-43 from the nucleus to the cytoplasm. (B) In another microscopic field of the same tissue section, neurons with cytoplasmic taupositive inclusions (arrows) maintain their nuclear TDP-43-immunoreactivity (100X). 
TABLE 1

Numbers of cases in each diagnostic category before and after TDP-43 immunohistochemistry, mean age at death, and progranulin mutation status

\begin{tabular}{lccc}
\hline & $\mathbf{n}$ & Mean age ( \pm SEM) & PGRN Mutations (+/-) \\
\hline FTLD-U & $68->67$ & $66 \pm 1.5$ & $14 / 35$ \\
FTLD-U, non-TDP-43 & $0->4$ & $57 \pm 4.5$ & $0 / 2$ \\
FTLD-U with VCP mut. & $2->2$ & $54 \pm 6.5$ & $0 / 2$ \\
DLDH & $4->1$ & 98 & $0 / 1$ \\
Other neurodegen. & $23->23$ & $74 \pm 2.5$ & $0 / 5$ \\
Controls & $12->12$ & $77 \pm 2.7$ & $0 / 2$ \\
\hline
\end{tabular}

PGRN, progranulin; FTLD-U, frontotemporal lobar degeneration with ubiquitinated inclusions; VCP mut., mutations in valosin-containing protein; DLDH, dementia lacking distinctive histopathology; neurodegen., neurodegenerative diseases 
TABLE 2

Hippocampus: Frequent TDP-43+ Dystrophic Neurites (DN) in CA1 are Associated with progranulin (PGRN) Mutations

\begin{tabular}{lcc}
\hline & Frequent DN & Absent to moderate DN \\
\hline $\mathrm{n}$ & $25(39 \%)$ & $39(61 \%)$ \\
Age ( $\mathrm{SEM})$ & $67 \pm 1.7$ & $66 \pm 1.9$ \\
Male/female & $13 / 12$ & $21 / 18$ \\
Family history (+/-) & $12 / 10$ & $14 / 19$ \\
PGRN Mutation (+/-) & $9 / 6 * *$ & $5 / 24$ \\
MND (+/-) & $1 / 24$ & $6 / 33$ \\
Solid CI predominant (+/-) & $12 / 11$ & $25 / 12$ \\
Language presentation (+/-) & $4 / 17$ & $7 / 25$ \\
\end{tabular}

** $\mathrm{p}=0.007$

MND, motor neuron disease; CI, cytoplasmic inclusions in the dentate gyrus 
TABLE 3

Frontal Cortex: Frequent Long TDP-43+Dystrophic Neurites (DN) are Associated with Progranulin (PGRN) Mutations

\begin{tabular}{|c|c|c|}
\hline & Frequent long DN & Absent to moderate long DN \\
\hline $\mathrm{n}$ & $22(35 \%)$ & $41(65 \%)$ \\
\hline Age $( \pm$ SEM $)$ & $66 \pm 1.8$ & $66 \pm 1.6$ \\
\hline Male/female & $12 / 10$ & $22 / 19$ \\
\hline Family history (+/-) & $12 / 7^{*}$ & $11 / 25$ \\
\hline PGRN mutation (+/-) & $10 / 4^{* * *}$ & $3 / 27$ \\
\hline $\operatorname{MND}(+/-)$ & $0 / 22$ & $6 / 35$ \\
\hline Solid CI predominant $(+/-)$ & $20 / 0^{* *}$ & $19 / 15$ \\
\hline Language presentation $(+/-)$ & $5 / 15$ & $7 / 26$ \\
\hline \multicolumn{3}{|l|}{$* \mathrm{p}=0.02$} \\
\hline \multicolumn{3}{|l|}{ ** $\mathrm{p}=0.0003$} \\
\hline$* * * * 0.00008$ & & \\
\hline
\end{tabular}


TABLE 4

Frontal cortex: Frequent Dot-like TDP-43+ Dystrophic Neurites (DN) are not Associated with Progranulin (PGRN) Mutations

\begin{tabular}{lcc}
\hline & Frequent dot-like DN & Absent to moderate dot-like DN \\
\hline $\mathrm{n}$ & $9(14 \%)$ & $54(86 \%)$ \\
Age ( $\mathrm{SEM})$ & $59 \pm 2.4^{*}$ & $67 \pm 1.3$ \\
Male/female & $5 / 4$ & $29 / 25$ \\
Family history (+/-) & $3 / 5$ & $20 / 27$ \\
PGRN mutation (+/-) & $1 / 5$ & $12 / 26$ \\
MND (+/-) & $2 / 7$ & $4 / 50$ \\
Solid CI predominant $(+/-)$ & $3 / 6 *$ & $36 / 9$ \\
Language presentation $(+/-)$ & $0 / 7$ & $12 / 34$ \\
\hline$* 0.01$ & &
\end{tabular}

MND, motor neuron disease; CI, cytoplasmic inclusions in the frontal cortex 
TABLE 6

Number of FTLD-U cases with hippocampal sclerosis and frequent CA1 dystrophic neurites in each frontal cortex pathology subtype

\begin{tabular}{|c|c|c|c|}
\hline & \multicolumn{2}{|c|}{ Number of cases with hippocampal sclerosis (/total examined) } & \multirow[b]{2}{*}{$\begin{array}{l}\text { Cases with frequent } \\
\text { DN in CA1 (/total } \\
\text { examined) }\end{array}$} \\
\hline & Posterior hippocampus & Anterior hippocampus & \\
\hline Unclassifiable & $4 / 9$ & $3 / 5$ & $1 / 8$ \\
\hline No frontal pathology & $2 / 2$ & $0 / 0$ & $0 / 2$ \\
\hline Type 1 & $3 / 13$ & $2 / 7$ & $0 / 13$ \\
\hline Type 2 & $2 / 12$ & $4 / 5$ & $4 / 12$ \\
\hline Type 3 & $5 / 27$ & $8 / 13$ & $21 / 26 * * *$ \\
\hline Type 4 & $1 / 2$ & $0 / 0$ & $0 / 2$ \\
\hline Total & $17 / 69(25 \%)$ & $17 / 30(57 \%)^{* *}$ & $26 / 61(43 \%)$ \\
\hline
\end{tabular}




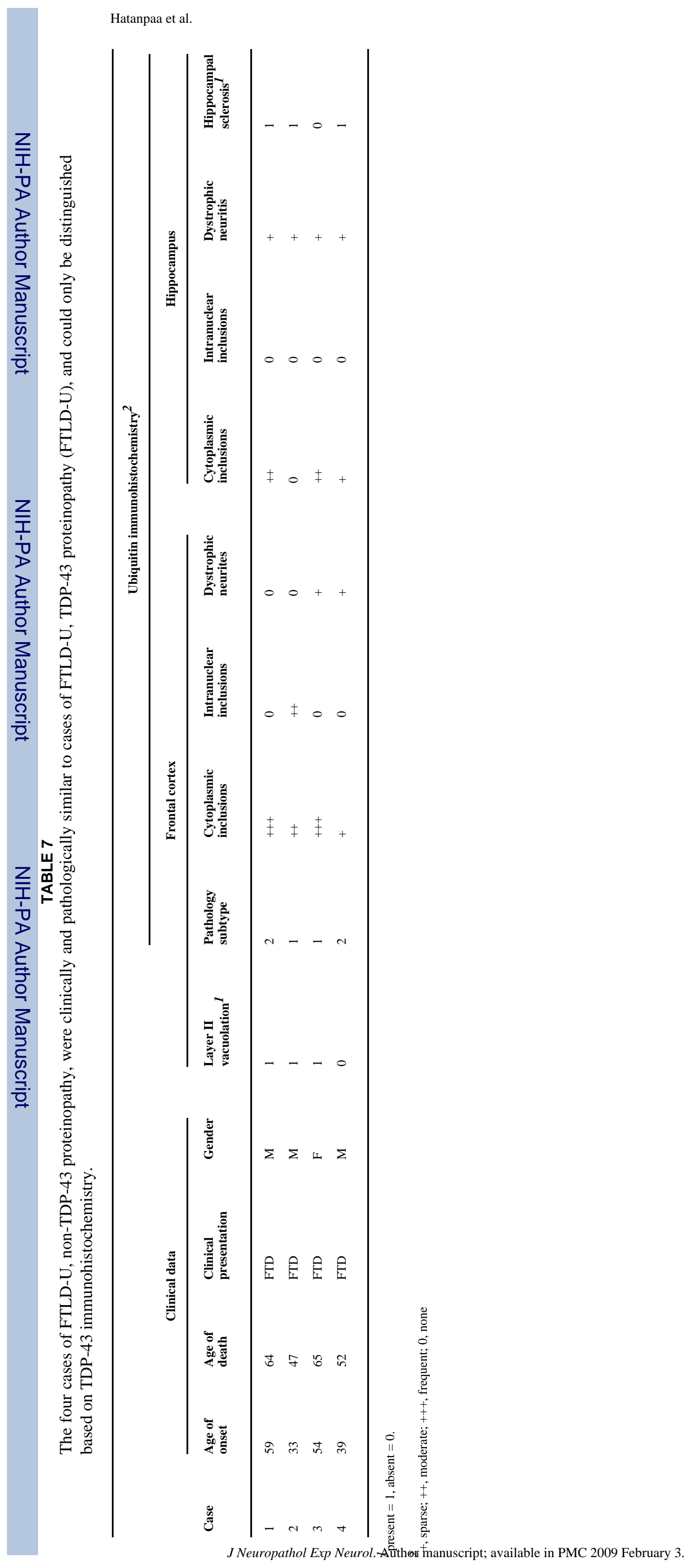

Page 23 